\title{
Prolong the Lifetime of Wireless Sensor Network using Fuzzy Logic to Control the Movement of the Mobile Sink
}

\author{
Mostafa Noorikhameneh \\ Department Computer Engineering \\ Ashtian Branch Islamic Azad University \\ Ashtian, Iran
}

\author{
Morteza Moosavi \\ Department Computer Engineering \\ Arak Branch Islamic Azad University \\ Arak,Iran
}

\begin{abstract}
Wireless sensor network is a wireless network which includes some sensor nodes. The sensor nodes are as the same and limited battery power. Wireless sensors could monitor physical or environmental conditions of a region. Aggregation data by sensors transmit to a sink. Because of limited power battery of sensor nodes, sending data from sensors to sink is a challengeable issue in these networks. focus in this paper proposed two algorithms for send data from sensor node to the sink. In first algorithm was utilized mobile sink and clustering such that design a paths for mobile sink by fuzzy logic which this paths include all of cluster head. In second algorithm sink path is fixed and for sending data from sensor cluster heads to the sink was utilize from Rendezvous Nodes. In this method selection of cluster head and Rendezvous Nodes was used by fuzzy logic
\end{abstract}

Then the simulation of proposed method using the network simulator OMNeT++ useful for debugging, performance evaluation, the simulation results show that proposed method gives better average energy, throughput and energy consumption and effectively Reduction delay.

\section{Keywords}

Wireless sensor network, mobile sink, clustering, Rendezvous Nodes, fuzzy logic, routing

\section{INTRODUCTION}

A wireless sensor network (WSN) comprises spatially distributed autonomous devices using sensors to cooperatively monitor physical or environmental conditions, such as temperature, sound, vibration, pressure, motion etc. at different locations. Subsequent to collaborative sensing of a given environment, the nodes perform in-network computation and communicate with a base station when a targeted event takes place [1]. A WSN has a number of exclusive characteristics when compared with conventional wireless networks. These include limited bandwidth, limited computation capability of individual nodes, and limited energy supply. Self-organization, dynamic network topology, and multi-hop routing are additional key possible features of a WSN, which make them important for many applications. It is advantageous to perform precise simulations or to develop models before deploying WSNs in the field. This is because WSNs may be deployed randomly in an ad-hoc manner with a large number of tiny nodes. Simulations help in the validation and evaluation of the performance of sensor networks within certain application environments, something that was not possible to achieve a number of years ago. Consequently, simulation of sensor networks is therefore gaining greater demand because of their capabilities, lower energy constraints and the use of a larger number of nodes compared to conventional wireless networks.

In addition, the structure of network topology is very complicated and the network topology changes quickly, WSN faces lots of challenges in practical applications. As the sensor, nodes are supplied with energy by batteries of limited energy and it is difficult to charge and replace, thus, resulting in the node is abnormal working. The whole network may be interrupted because one or more nodes stop working. A wsn normally consists of many inexpensive wireless nodes, each capable of collecting, processing, and storing environmental information, and communicating with neighboring nodes. Many WSN applications have been developed, such as emergency guiding [2, 3], object tracking [4, 5], and environment monitoring [6,7]. And many researchers are currently engaged in developing schemes that fulfill these requirements. Figure (1) shows the wireless sensor network (WSN).

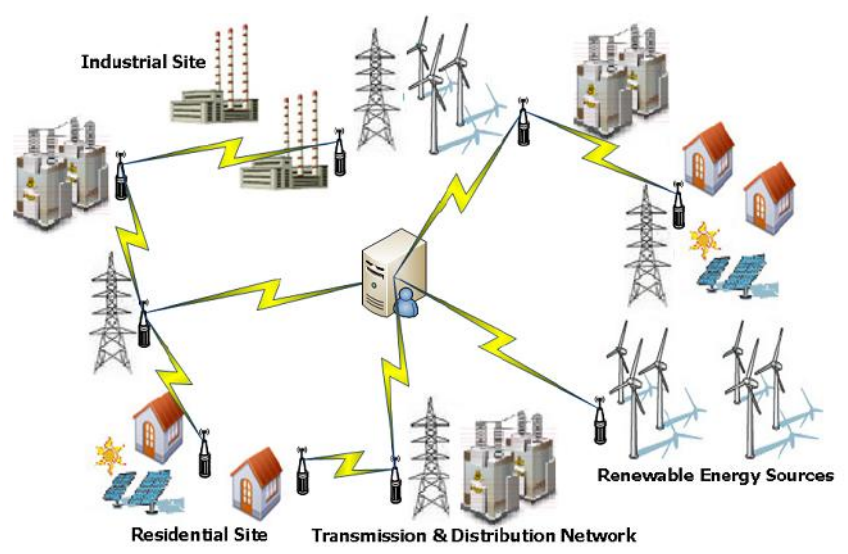

Figure 1. An example of wireless sensor network (WSN)

The propose of this research in this paper, Prolong the Lifetime of Wireless Sensor Network Using Fuzzy Logic to Control the Movement of the Mobile Sink;

The main focus on this subject proposed two algorithms for send data from sensor node to the sink. In first algorithm was utilized mobile sink and clustering such that design a paths for mobile sink by fuzzy logic which this paths include all of cluster head. In second algorithm sink path is fixed and for sending data from sensor cluster heads to the sink was utilize from Rendezvous Nodes. In this method, selection of cluster head and Rendezvous Nodes was used by fuzzy logic.

The paper is structured as follows. Section II, related work. Section, II routing protocol in WSN. In section IV, proposed method. In section V, Simulation result. Finally, concluding remarks are given in section VI.

\section{RELATED WORK}

Some previous works $[8,9,10,11,12]$ propose low-latency converge cast algorithms for wireless sensor networks. The work [13] proposes an energy-efficient and low-latency MAC, called DMAC, for converge cast. The authors design a scheduling method to stagger the wake-up times of sensors to facilitate packet delivery to the sink. The work [14] introduces 
a TDMA schedule to minimize the time needed for converge cast. The authors reduce the tree-based converge cast problem to a multiple routing paths problem. A distributed algorithm is proposed to schedule the transmission time of each routing path (from each node to the sink) to achieve low latency converge cast.

The authors in [15] observes that balanced tree can have the benefits of reducing report latency. They propose tree construction algorithm, which runs in a level-by-level fashion. For those nodes in a level, the algorithm utilizes the concept of bipartite graph to balance the number of their child nodes. After forming the tree, the proposed link scheduling method an applied to achieve low-latency, converge cast. In LEACH [16], the authors discuss an energy efficient algorithm. Various algorithms developed after that is based on this algorithm. In order to determine the cluster head, LEACH uses randomization technique. In case of the discussed algorithm, after each cycle or after certain time interval, sensors elect themselves to be local cluster - heads, with a certain probability. Subsequently, the status of these cluster heads is broadcasted to the other sensors in the network. Next, each sensor node determines to which cluster it wants to belong. This is done by selecting the cluster - head for which minimum communication energy is required. In the initial stage of LEACH-C [17] algorithm, each node sends information to the base station. The information is about current location and energy level of that node. An optimization algorithm is run by the base station to determine the clusters for that round. Hence, this algorithm necessitates the position of each node at the beginning of each round. This necessitates a global positioning system, GPS. The average node energy is calculated by the base station. The nodes having energy below this average cannot become cluster heads for the current round. In order to determine the best nodes to be cluster heads for the next round and the associated clusters, the base station uses the remaining nodes as possible cluster heads and runs an algorithm known as simulated annealing algorithm, which is based on thermodynamics principles. In sparse sensor networks where the path is random [18], the mobile sinks are often mounted on some people or animals to collect interested information sensed by the sensors. However, latency is increased because a sensor has to wait for a mobile sink before its data can be delivered. Liu et al. in [19] proposes a proactive data reporting protocol, Sink Trail, which achieves energy efficient data forwarding to multiple mobile sinks with broadcasting sink location messages. However, this kind of data collection schemes is undesirable, especially when the sensor network scale increases, as frequent message flooding will cause serious congestion in network communication and significantly impair the sensor network lifetime.

\section{ROUTING PROTOCOL IN WSN}

Having looked at the challenges in the wireless sensor networks it is time to dive in to the routing protocols. Since it is difficult for a single protocol to solve all the above challenges the routing protocols were designed with specific goals. In this paper used Low-energy adaptive clustering hierarchy $(\mathrm{LEACH})$ based on their goals in this paper

\subsection{Low-energy adaptive clustering hierarchy (LEACH)}

LEACH $[20,21]$ is the first and most popular energy-efficient hierarchical clustering algorithm for WSNs that was proposed for reducing power consumption. In LEACH, the clustering task is rotated among the nodes, based on duration. Each cluster head $(\mathrm{CH})$ to forward the data to the base station (BS) uses direct communication. It uses clusters to prolong the life of the wireless sensor network. LEACH is based on an aggregation (or fusion) technique that combines or aggregates the original data into a smaller size of data that carry only meaningful information to all individual sensors. LEACH divides the a network into several cluster of sensors, which are constructed by using localized coordination and control not only to reduce the amount of data that are transmitted to the sink, but also to make routing and data dissemination more scalable and robust. LEACH uses a randomize rotation of highenergy $\mathrm{CH}$ position rather than selecting in static manner, to give a chance to all sensors to act as $\mathrm{CHs}$ and avoid the battery depletion of an individual sensor and dyeing quickly. The operation of LEACH is divided into rounds having two phases each namely (1) a setup phase to organize the network into clusters, $\mathrm{CH}$ advertisement, and transmission schedule creation and (2) a steady-state phase for data aggregation, compression, and transmission to the sink.

LEACH is completely distributed and requires no global knowledge of network. It reduces energy consumption by (a) minimizing the communication cost between sensors and their cluster heads and (b) turning off non-head nodes as much as possible. LEACH uses single-hop routing where each node can transmit directly to the cluster-head and the sink. Therefore, it is not applicable to networks deployed in large regions. Furthermore, the idea of dynamic clustering brings extra overhead, e.g. head changes, advertisements etc., which may diminish the gain in energy consumption. While LEACH helps the sensors within their cluster dissipate their energy slowly, the CHs consume a larger amount of energy when they are located farther away from the sink. In addition, LEACH clustering terminates in a finite number of iterations, but does not guarantee good $\mathrm{CH}$ distribution and assumes uniform energy consumption for $\mathrm{CHs}$.

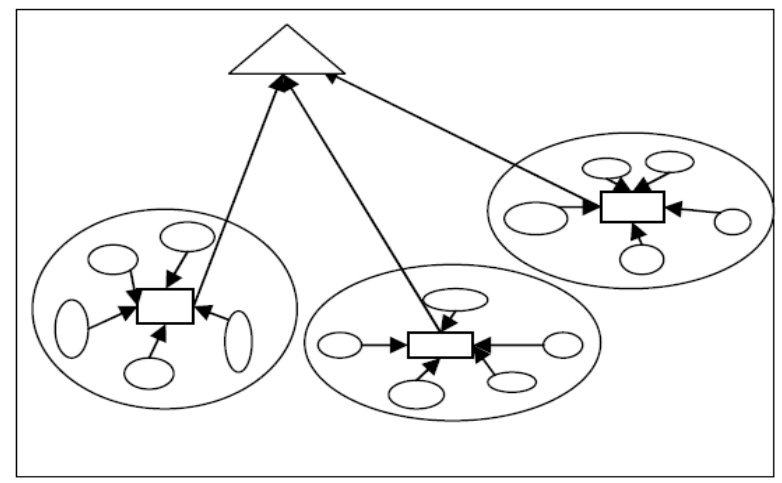

Figure 2. LEACH Frameworks

\section{PROPOSED METHOD}

\subsection{Network model and assumptions}

Wireless sensor network deployment environment is a twodimensional flat area, and the distance metric in this network is the Euclidean metric. Therefore, in order to calculate the distance between two nodes in the network, the proposed method use the formula of calculating the Euclidean distance. It is also assume that a system consists of a number of sensor nodes that are randomly deployed in the study area. The sensor nodes are fixed. This means that the nodes cannot move. Each node has the primary energy of $E_{0}$. There is also a moving sink (MS) in the network that can move in the network and receive the data that is collected by the sensor nodes. It also assume that the energy of the moving sink is infinite. The other assumption is that each sensor node is equipped with GPS system; therefore, each node is aware of its geographical location. 


\subsection{First proposed method: (Mobile sink fuzzy)}

The first proposed method, which is called as MSF, is similar to LEACH protocol, is divided into several occasions. On each turn, each node generates and sends a set of data with a fixed rate. In MSF method, each turn consists of three phases.

\subsubsection{First phase (installation)}

consist of three stages. In the first stage, normal nodes (NN) and cluster head nodes $(\mathrm{CH})$ are specified. The second phase is the formation of clusters, and Scheduling is done at the end so that $\mathrm{CH}$ sends a scheduling to each of the nodes belonging to its cluster.

\subsubsection{Determination stage of $\mathrm{CH}$}

At this stage, at this time of the implementation, each node decides whether to be a $\mathrm{CH}$ or not. This decision is done on the basis of two criteria. The first criterion is the permitted percentage of nodes that are allowed to become a $\mathrm{CH}$. For example, for a definite network it is said that $5 \%$ of the total number of nodes on the network must be converted to $\mathrm{CH}$, for instance. The second criterion is the number of times a node has been converted to $\mathrm{CH}$. The decision is done for a node such as node $\mathrm{n}$ with a random number generated between 1 and 0 . The threshold is calculated according to equation (1):

$$
=\left\{\begin{array}{l}
\frac{P}{1-P *\left(r \bmod \frac{1}{P}\right)} \quad \text { if } n \in G \\
0 \quad \text { otherwise }
\end{array}\right.
$$

Where $\mathrm{P}$ is the permitted percentage of heads, $\mathrm{r}$ is the present time and $\mathrm{G}$ is the sum of nodes that have not been converted into cluster head node in the last $\frac{1}{P}$ yet.

In this paper, the nodes can use the distance to select their head node. This means that each node estimates its distance from the head nodes and selects the closer head node as its head node. If some $\mathrm{CHs}$ are in the same distance from a node, selection is done randomly.

\subsubsection{The stage of determining the categories}

As the node decided to which category it belongs, the node must notify the leader of this issue. Each node does this issue by retransmitting the data to the selected head.

\subsubsection{The scheduling stage}

The head node does a scheduling based on the number of nodes belonging to a scheduling and sends back this scheduling to the member nodes in the form of a message.

Second phase (Information transfer) of information the nodes send their data to their cluster head

A flow chart is provided in Figure (3), which indicates the details of the first two phases.

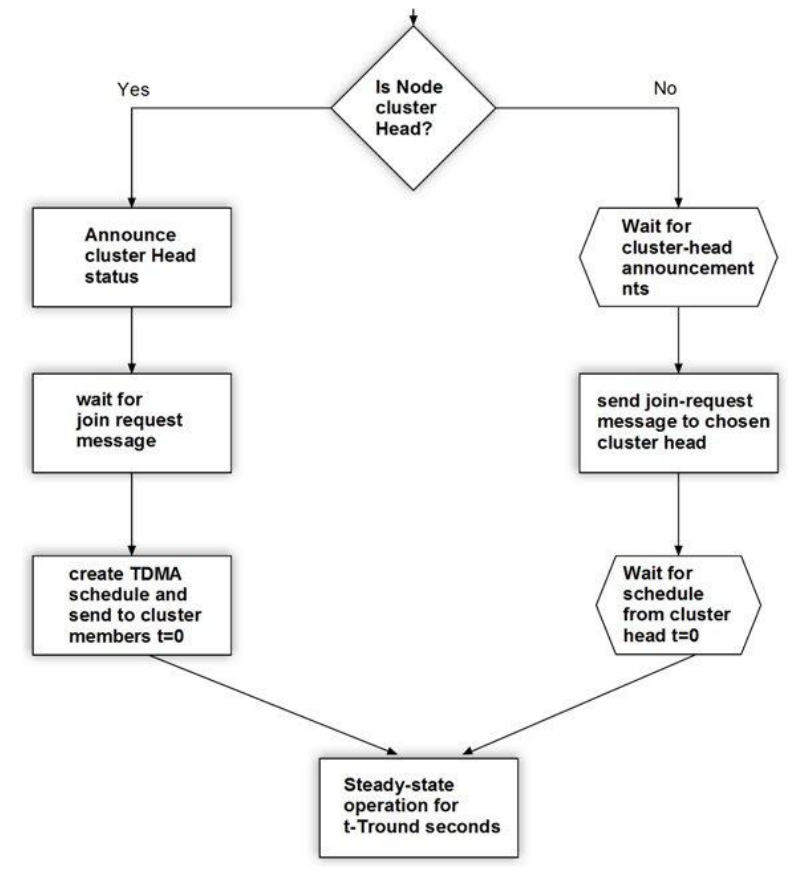

Figure 3. The flowchart of first phase and second phase [22]

In this paper, it also assume that the nodes are randomly deployed in the network; therefore, these routes may consume a lot of energy in the network and not minimize energy consumption. As a result, here it will need to have an intelligent algorithm to determine the route of the moving sink. The proposed method use fuzzy logic to determine the route of the moving sink and it is the moving sink that decides how to move in the network.

\subsubsection{Third phase (routing sink)}

The information is transferred to each moving sink (MS). In this phase, a route is selected for the moving sink using the Fuzzy Logic so that causes the moving ink to move close to the $\mathrm{CH}$ related to each cluster and collect the data in the network. The third phase runs after the end of the second phase. When the data transfer phase ended, the routing phase of the moving sink starts. At the end of the data, transfer phase when all the member nodes of the clusters send their data to their head, and the head node collects and compresses all data. A message with the name $\mathrm{CH}$ _Info that contains only the following information is sent to the sink:

1. Cluster head ID

2. Position of the $\mathrm{CH}$ in the network

3. The residual energy of the cluster head

Receiving the $\mathrm{CH}$ _Info messages, the sink obtains messages detailed information about the cluster nodes from the $\mathrm{CHs}$ and their position. The sink used in this method is a moving sink. The moving sink moves in the network and collects information from the cluster nodes. If the moving sink moves close to the cluster head nodes and collects their information, the cluster head nodes then transfer the gathered information to the moving sink through a shorter distance; therefore, they consume less energy.

\subsection{Using fuzzy logic for routing the Mobile Sink}

Input parameters of the fuzzy system should be related to the head node because the goal is to allocate a priority to the head nodes; therefore, several different parameters can be defined 
for a head node where the two following parameters are used among these parameters.

1. The residual energy of the head: the head with less energy has the high priority.

2. The distance from the moving sink: The head, which has a greater distance from the mobile sink, needs more energy to communicate with the mobile sink; therefore it has the higher priority.

\subsubsection{Fuzzy System}

In this paper, the fuzzy systems are "with fuzzification and defuzzification". Figure (4) indicates a block view of the fuzzy system.

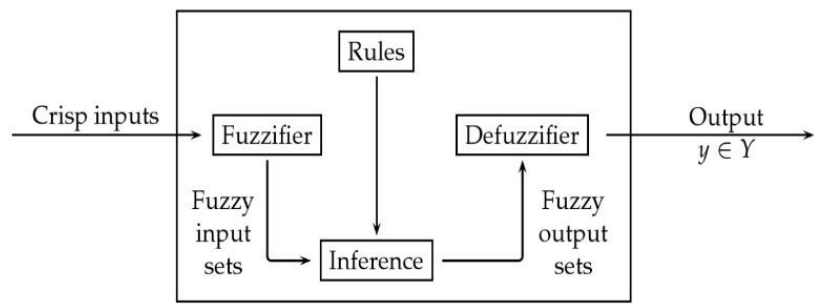

Figure (4). indicates a block view of the fuzzy system

In this fuzzy system, the inputs are first converted to a fuzzy set using a Fuzzifier, where the Fuzzifier does this using the input membership functions. The desired result is produced as a fuzzy set which the Inference parts of this work. This section covert the fuzzy input into the fuzzy output based on fuzzy rules of "if-then" and then the fuzzy output is converted into the numerical output using the Defuzzifier section (the Defuzzifier does this by the help of the output membership function which is determined in the next section).

The conducted inference mechanism in this system is based on Table (1) of the basic rules. This method includes two fuzzy set (distance and energy) where based on these two inputs, an output based on the table of basic rules is produced that is table (1).

Table 1. Basic rules for the fuzzy inference system

\begin{tabular}{|c|c|c|}
\hline Energy & distance & Priority \\
\hline Low & Near & Medium \\
\hline Low & Medium & High \\
\hline Low & Far & v-high \\
\hline Medium & Near & Low \\
\hline Medium & Medium & Medium \\
\hline Medium & Far & Low \\
\hline High & Near & v-low \\
\hline High & Medium & Low \\
\hline High & Far & Medium \\
\hline
\end{tabular}

\subsubsection{Membership functions for the fuzzy sets}

Variables of the residual energy and the distance from the sink are considered as the two fuzzy sets. the residual energy set select the following three sets 1) Low 2) Medium and 3) High. One-dimensional and triangular membership functions.

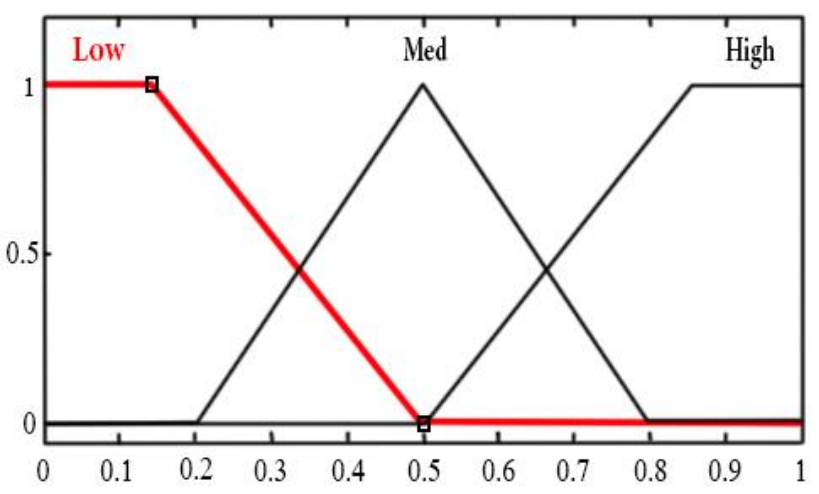

Figure 5. Membership functions for the subsets of the fuzzy set of the residual energy

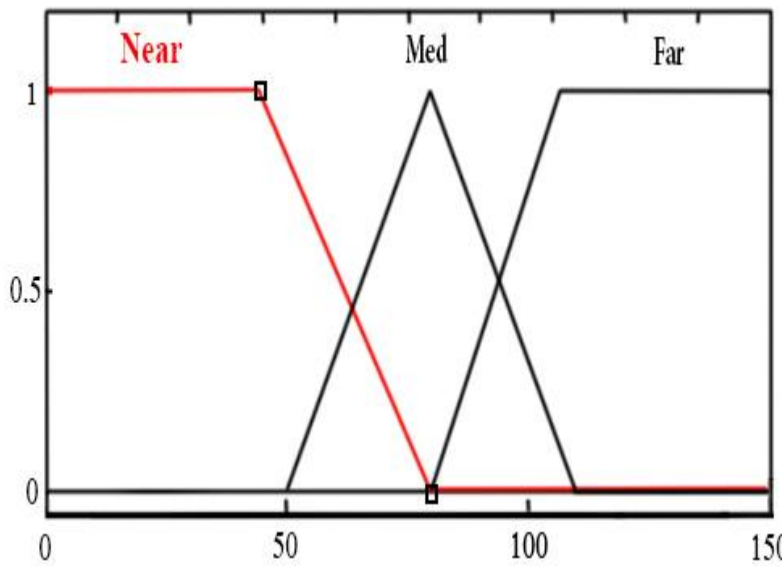

Figure 6. Membership functions for the subsets of the fuzzy set of distance from the sink

For the set of the distance from the sink, the fuzzy select the following subsets 1, near 2, Med and 3, Far.

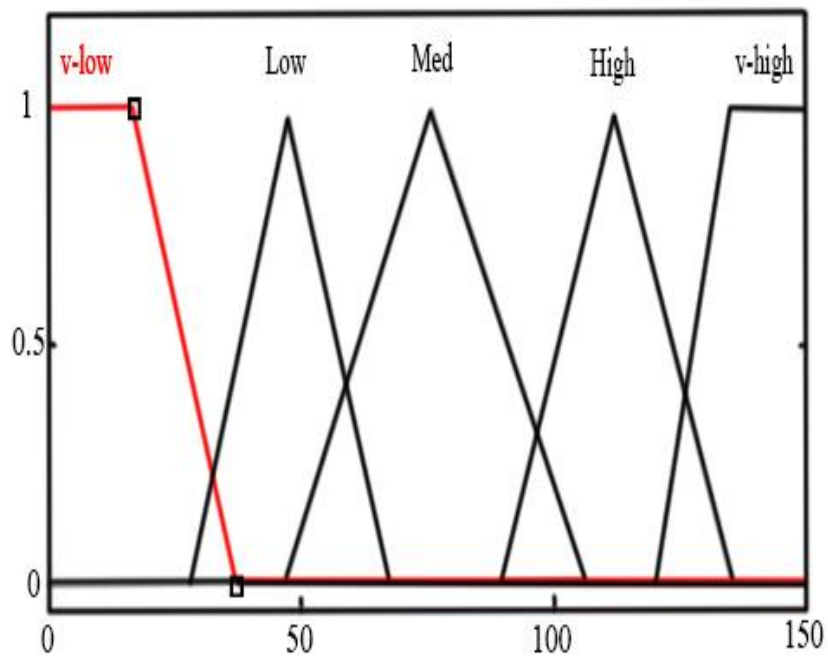

Figure 7. Membership functions for the subsets of the fuzzy set of priority

For the output fuzzy set, the priority of the fuzzy system select the subsets 1) v-low 2) low 3) Med 4) high 5) v-high. 


\section{Pseudo-code of MSF algorithm \\ 1- Each node compute $T(n)$ and generate random number $r$ \\ 2- If $r<T(n)$ then \\ i. node Type $=\mathrm{CH}$ \\ 3- else \\ i. node Type $=\mathrm{NN}$ \\ 4- if (node Type $=\mathrm{CH}$ ) then broadcast $\mathrm{CH} 2 \mathrm{NN}$ messages \\ 5- if (node Type $=\mathrm{NN}$ ) and (Received CH2NN message) then \\ i. Find nearest $\mathrm{CH}$ \\ ii. broadcast DetermineCH for nearest $\mathrm{CH}$ \\ 6- if (node Type $=\mathrm{CH}$ ) and $($ Received all DetermineCH messages) then send TDMA message to node members of it's cluster \\ 7- if $($ nodeType $=\mathrm{NN})$ and $($ Received TDMA message $)$ then send DataTo $\mathrm{CH}$ message according to TDMA messages \\ 8- all CHs send $\mathrm{CH}$ _Info to sink \\ 9- Sink received all $\mathrm{CH}$ _Info messges and save they data in PathList array \\ 10- Sink compute Priority for each $\mathrm{CH}$ then design path \\ 11- Sort PathList according to Priority \\ 12- Visit CHs with PathList info \\ 13- When reach the end of PathList then}

\section{Figure 8. Pseudo-code of MSF algorithm}

In this section, a pseudo code is expressed for the MSF algorithm. Indicates the operational pseudo code, which is run on the network at every turn. Suppose that round is the counter of turns and MAXRound is the highest number of rounds.

\subsection{Second proposed method (Clustering Base Fuzzy logic):}

The second proposed method introduced in this paper is a routing protocol based on fuzzy logic and clustering so that the fuzzy logic is used to determine the cluster heads. The second proposed method is then called as clustering based on fuzzy logic $(\mathrm{CBF})$.

$\mathrm{CBF}$ algorithm assume that there is a moving sink where the sink is moving on a predetermined route. Network area has been considered as a square. The route of the sink in this square area has been indicated as in Figure (9).

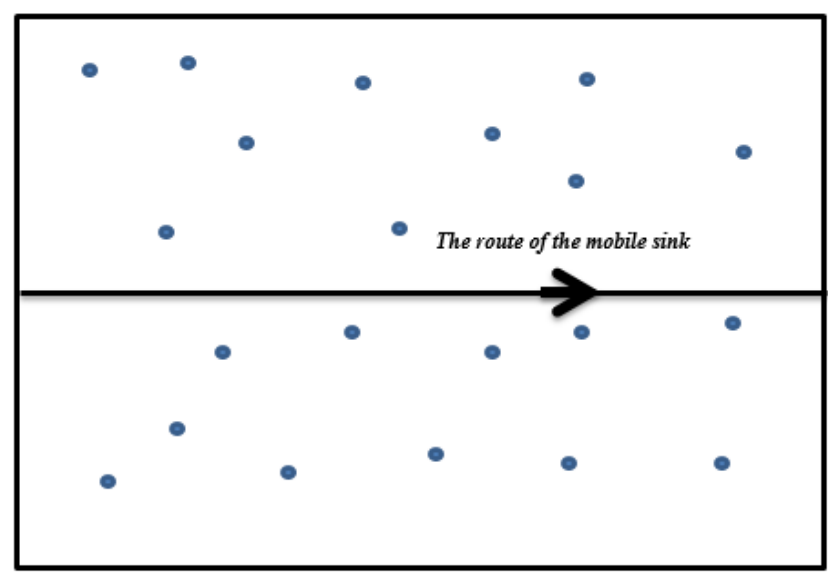

Figure 9. The network model for the CBF algorithms,

The line with a flash on it indicates the route of the moving sink in the network. The moving sink is commuting in this route. In addition, collect the data along the way. The details of the decision are stated next. The works that are done at every turn are as follows:

1. Determining the cluster heads using the fuzzy logic

2. The formation of clusters

3. The transmission of information to the cluster heads

4. The transmission of data from the cluster heads to the sink using the RN nodes

These steps are performed in sequence in each turn. In stage 1, the cluster heads are determined through fuzzy logic based on two parameters of energy and the distance from the sink. Then, they form the clusters and the information is transmitted from the sensors to the cluster heads and in the final phase, the information is transmitted to the sink from the cluster heads using the RN nodes. The formation stages of the clusters and transmission of data to the head clusters is the same as the MSF algorithm, which is described in the previous section.

\subsubsection{Determining the intersecting nodes using the} fuzzy logic:

Determining $\mathrm{RN}$ nodes is based on the following two parameters:

1. The distance of the node from the route of the moving sink

2. The number of the neighboring nodes

Nodes that are within a certain distance from the route of the moving sink (The specified distance is determined in the fourth chapter) send a Select_RN message to its neighbors and then, each node obtains its neighbors by counting the number of Select_RN received messages.

As each node obtained its neighbors, uses a fuzzy system, through this fuzzy system the input of which is the number of the neighbors of the node and the distance from the route of the moving sink. An input is produced. If the output is greater than the threshold $\alpha$, the node is converted into an $\mathrm{RN}$ node; otherwise, the node continues to function normally. Using the threshold $\alpha$ value can control the number of $\mathrm{RN}$ nodes, and obtain the optimal number of $\mathrm{RN}$ nodes. 


\subsubsection{The fuzzy system to select the intersecting} nodes:

The input and output of this fuzzy system in this section is to determine the different $\mathrm{RN}$ nodes. To determine the RN nodes, the two parameters are used:

1. The distance of the node from the route of the moving sink: any node in a far distance from the route of the moving sink should have a low priority for being RN.

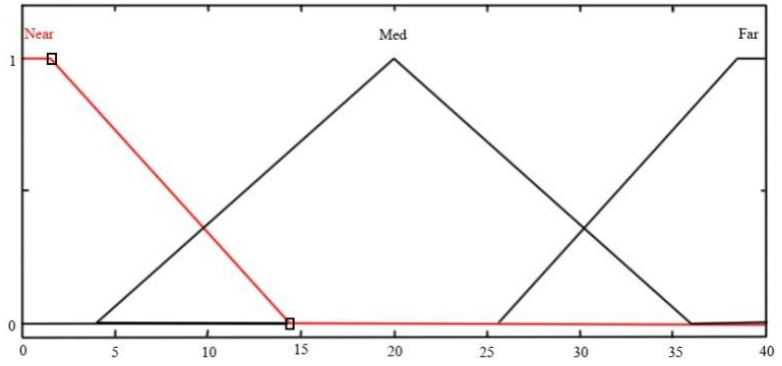

Figure 10. Membership functions for subsets of fuzzy set of the distance from sink

The fuzzy system consider the variable of distance from the route of the moving sink as a fuzzy set, which includes the following sets of Near, Med and Far.

Figure (10) and Figure (6) indicates the triangular membership functions for fuzzy sets of the fuzzy set of the distance from the route of the moving sink.

2. The number of node neighbors: any node, which has a high number of neighbors, is a better choice for being $\mathrm{RN}$, therefore it must have a higher priority.

The variable of the number of node's neighbors is considered as a fuzzy set, the number of neighbors of each node in the range $[0, \mathrm{n}]$ could be. where $\mathrm{n}$ is equal to the number of nodes on the network,

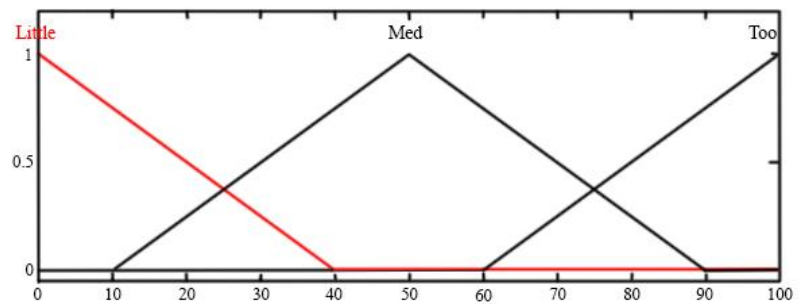

Figure 11. Membership functions for subsets of fuzzy set of the number of neighbors

This fuzzy set includes the following subsets of Too, Med and Little.

Table 2. basic rules for fuzzy inference system for selecting RNs

\begin{tabular}{|c|c|c|}
\hline $\begin{array}{c}\text { The number of } \\
\text { neighbors }\end{array}$ & Distance & Output \\
\hline Too & Near & v-high \\
\hline Too & Medium & High \\
\hline Too & Far & Medium \\
\hline Medium & Near & Low \\
\hline Medium & Medium & Medium \\
\hline Medium & Far & Low \\
\hline Little & Near & Medium \\
\hline Little & Medium & Low \\
\hline Little & Far & v-low \\
\hline
\end{tabular}

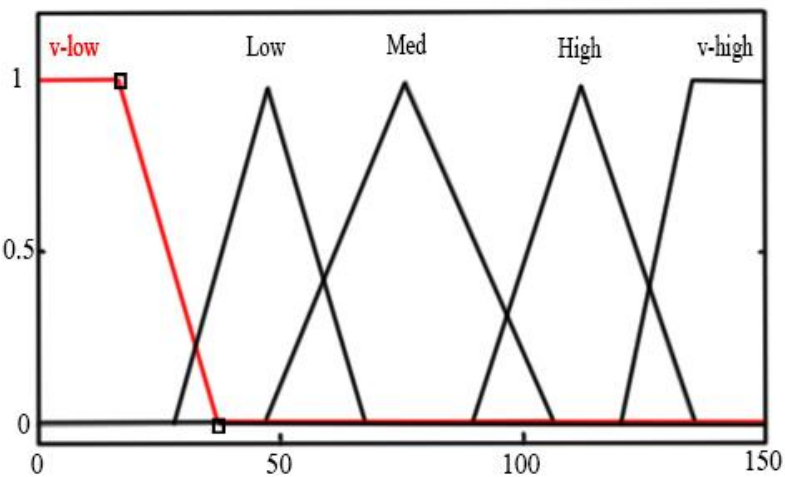

Figure 12. Membership functions for subsets of fuzzy set of priority for $\mathrm{RN}$ nodes

For the output fuzzy set, the priority of the fuzzy system select the subsets of 1) v-low 2) low 3) Med 4) high 5) v-high. And the membership functions are triangular.

\subsubsection{Determining the cluster heads using the fuzzy} logic

- The fuzzy logic is used in the CBF algorithm to select the cluster heads.

- Member nodes of the cluster use a fuzzy system to calculate this priority the input of which consists of the following two parameters:

\section{The residual energy of the node:}

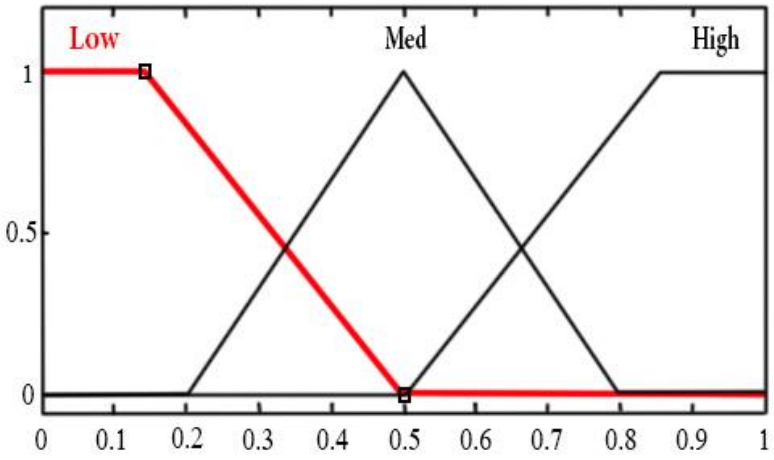

Figure 13. Membership functions for subsets of fuzzy set related to the residual energy of the node

Includes three subsets of 1) Low 2) Medium and 3) High. For each of these subsets, the fuzzy define a membership function. Membership functions are one-dimensional and triangular

The distance from the route of the moving sink:

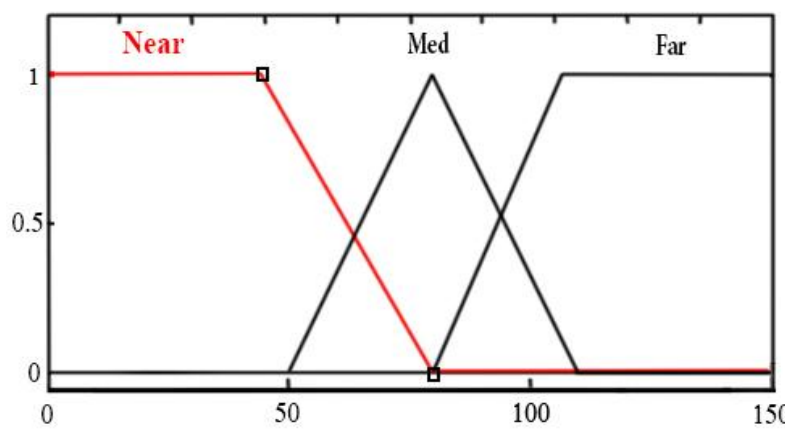

Figure 14 Membership functions for subsets of fuzzy set of the distance from the sink 
Includes the following subsets of Near, Med and Far

Table 3. Basic rules for fuzzy inference system to select cluster heads

\begin{tabular}{|c|c|c|}
\hline Energy & distance & Priority \\
\hline Low & Near & v-high \\
\hline Low & Medium & High \\
\hline Low & Far & Medium \\
\hline Medium & Near & Low \\
\hline Medium & Medium & Medium \\
\hline Medium & Far & Low \\
\hline High & Near & Medium \\
\hline High & Medium & Low \\
\hline High & Far & v-low \\
\hline
\end{tabular}

Table 3. Indicates the fuzzy rules related to the fuzzy system.

\section{Pseudo-code of the CBF algorithm}

1- If (round $==1$ )

i. Select RNs according to fuzzy logic base on distance and Count Neighbor

ii. Each node compute $T(n)$ and generate random number $r$

iii. If $r<T(n)$ then

$$
\text { i. } \quad \text { nodeType }=\mathrm{CH}
$$

iv. else

$$
\text { i. } \quad \text { nodeType }=\mathrm{NN}
$$

2- if (round != 1)

i. Select node with maximum priority (from previous round)

3- if (nodeType $=\mathrm{CH}$ ) then broadcast $\mathrm{CH} 2 \mathrm{NN}$ messages

4- if (nodeType $=\mathrm{NN}$ ) and $($ Received a $\mathrm{CH} 2 \mathrm{NN}$ message) then

i. Find nearest $\mathrm{CH}$

ii. Compute Priority according to fuzzy logic base on distance and energy

iii. broadcast DetermineCH for nearest $\mathrm{CH}$

5- if (nodeType $=\mathrm{CH})$ and $($ Received all DetermineCH messages) then

i. Select one neighbor with maximum Priority

ii. send TDMA and Priority message to node members of it's cluster

6- if (nodeType $=\mathrm{NN})$ and (Received TDMA) then send DataToCH according to TDMA messages
7- If (nodeType $==\mathrm{CH}$ ) and (Received all DataToCH messages) then

i. If sink is in it's range then send Data to sink direct ii. Else send data to nearest $\mathrm{RN}$ nodes

8- If (nodeType $==\mathrm{RN}$ ) and (Received Data message) then Send data to sink

9- $\quad$ round ++

10- If( round > MAXIMUM_ROUND) then End Simulation

Figure 15. Pseudo-code of the CBF algorithm

\section{SIMULATION RESULT}

This section includes simulation and evaluation of accurate proposed method. This simulation proved by OMNeT++, OMNeT++[1] (Objective Modular Network Testbed in C++) ist ein Simulations-Framework, mit dem Rechnernetze und Netzwerkprotokolle simuliert und Leistungsanalysen durchgeführt werden können. show in table 4 the metrics used to evaluate the performance are given below.

\begin{tabular}{|c|c|c|}
\hline parameter & Value & Description \\
\hline$x_{m}$ & $350 \mathrm{~m}$ & Area \\
\hline$\overline{y_{m}}$ & $350 \mathrm{~m}$ & Area Simulation \\
\hline$E_{0}$ & $0.3 \mathrm{~J}$ & Initial energy per node \\
\hline$\overline{E_{T x}}$ & $50 \mathrm{~nJ} / \mathrm{bit}$ & $\begin{array}{l}\text { Energy consumption for data } \\
\text { transfer in radio contact }\end{array}$ \\
\hline$E_{R x}$ & $50 \mathrm{~nJ} / \mathrm{bit}$ & $\begin{array}{l}\text { Energy consumption for data } \\
\text { transfer in radio contact }\end{array}$ \\
\hline$E_{D A}$ & $\begin{array}{c}5 \\
\mathrm{~nJ} / \text { bit/signal }\end{array}$ & $\begin{array}{l}\text { Energy consumption to collect } \\
\text { information. }\end{array}$ \\
\hline$E_{\text {amp }}$ & $\begin{array}{c}0.0013 \\
\mathrm{pJ} / \mathrm{bit} / \mathrm{m}^{4}\end{array}$ & $\begin{array}{c}\text { The energy expended by } \\
\text { amplifier In the case of multi } \\
\text { route }\end{array}$ \\
\hline$E_{f s}$ & $\begin{array}{c}10 \\
\mathrm{pJ} / \mathrm{bit} / \mathrm{m}^{2}\end{array}$ & $\begin{array}{l}\text { The energy expended by } \\
\text { Amplifier In the case of free } \\
\text { space }\end{array}$ \\
\hline $\mathrm{R}$ & $20 \mathrm{~m}$ & Radio range of each node \\
\hline $\mathrm{P}$ & $5 \%$ & $\begin{array}{l}\text { Percentage nodes to become } \\
\text { leader of the node }\end{array}$ \\
\hline Node & 100 & $\begin{array}{l}\text { Number of nodes on the } \\
\text { network. }\end{array}$ \\
\hline
\end{tabular}

Table 4. Simulation Parametrs

\subsection{Number of Dead Nodes}

Number of Dead Nodes: If one encounters a persistently DEAD node with error messages in the log "Node is DEAD. Dropping message." and there is no data being received from the node and/or the node does not respond to commands.

\subsection{Avreage energy}

The amount of energy in the network due to resource constraints such as power, energy, bandwidth, processing capabilities and storage space in the node, reducing routing 
overhead and ensure high rate packet delivery an important issue in wireless sensor networks.

$$
\operatorname{avgEnergy}=\frac{\text { RemainEnergy }(r)}{(100-\operatorname{dead}(r))}=\frac{\sum_{n=1}^{100} E(n)}{100-\operatorname{dead}(r)}
$$

\subsection{Delay}

In wireless sensor networks, End-to-end delay refers to the time taken for a packet to be transmitted Around the Network from source to destination.

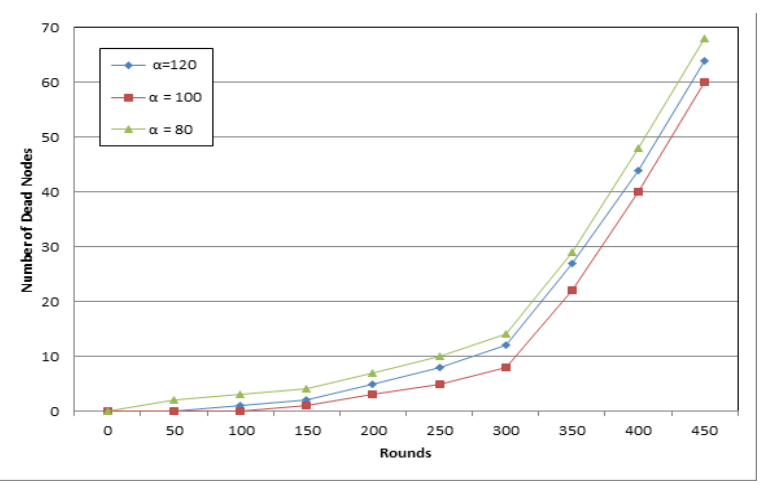

Figure 16. Number of dead nodes VS CBF algorithm

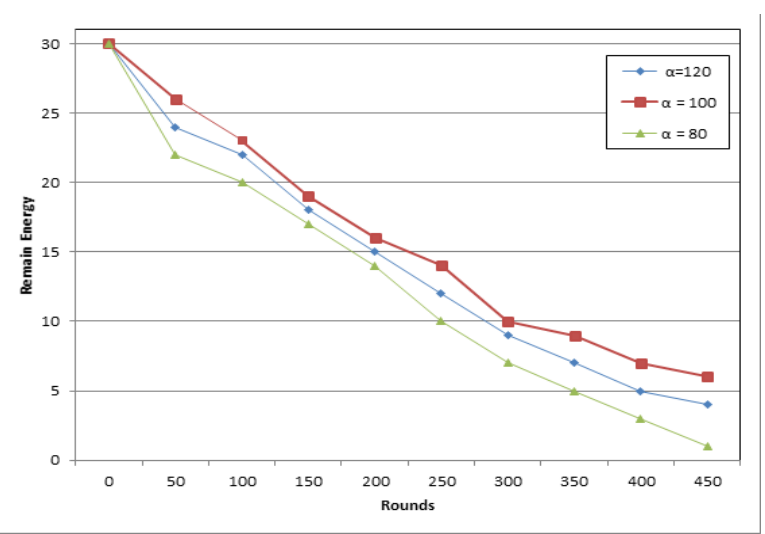

Figure 17. Residual energy in CBF algorithm

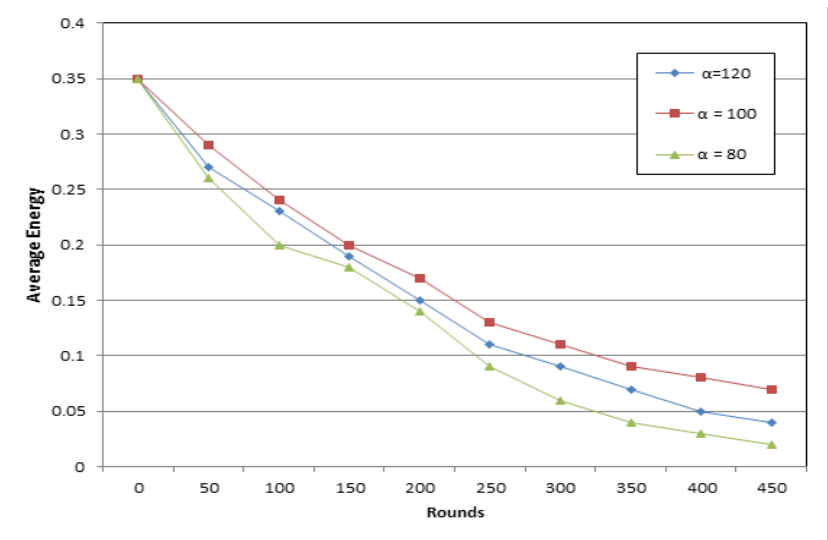

Figure 18. Average energy nodes Vs CBF algorithm

Figure (16), (17) and (18) show that the A proposed algorithm using Fuzzy Logic to Control the Movement of the Mobile Sink. To find the optimal value for the parameter $\alpha$, three levels of choice and value for the simulation value calculated with these three wishes The results indicate a near-optimal value for the parameter $\alpha$. Values for this parameter is considered equal to 120,100 and 80 respectively.
Value 100 for $\mathrm{CBF}$ is a good choice for the parameter $\alpha$ in the algorithm.

Also the proposed algorithm CBF performs better, extends several rounds more than the other algorithm for data transmission, and saves more energy.

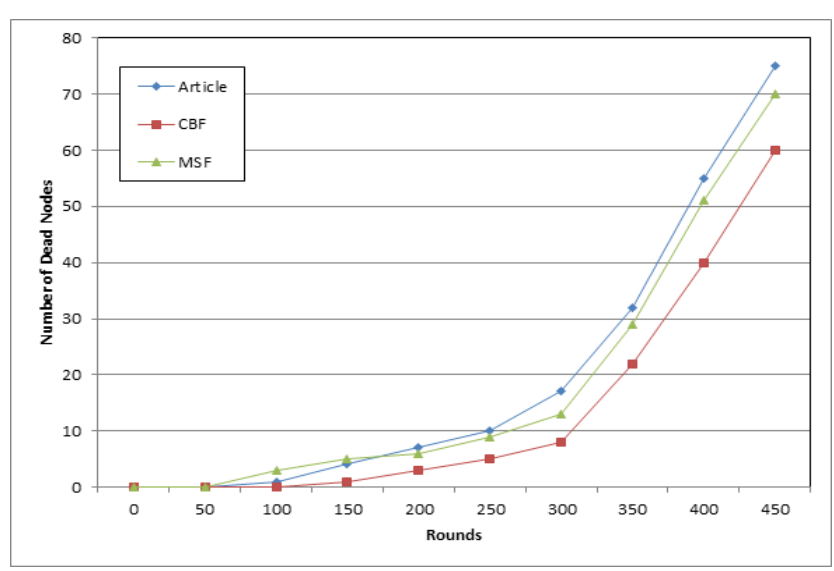

Figure 19. Comparison dead node Vs Round

Figure (19) Show that the number of dead nodes in different in $\mathrm{CBF}$ algorithm is lower

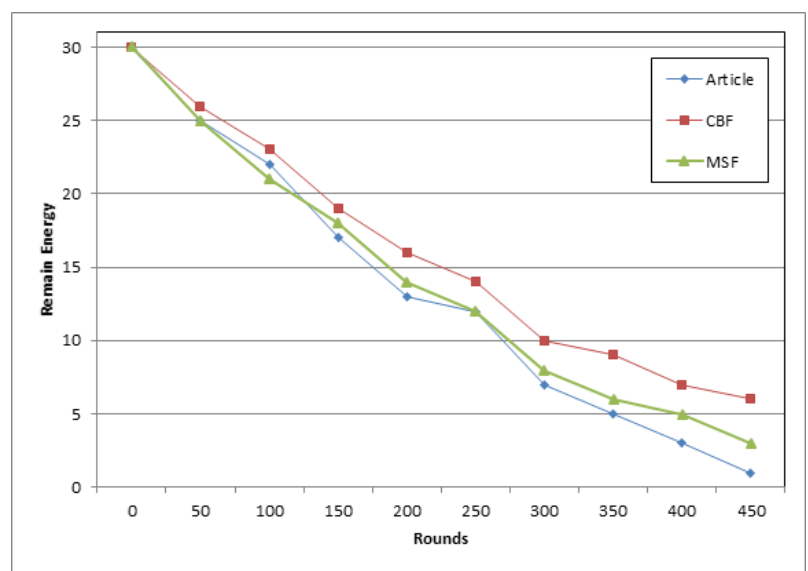

Figure 20. Comparison Remaining energy Vs three algorithm

Figure (20). Show that the using Fuzzy Logic for node selected in cluster is performance better. Proposed algorithm CBF in Comparison MSF is better performance.

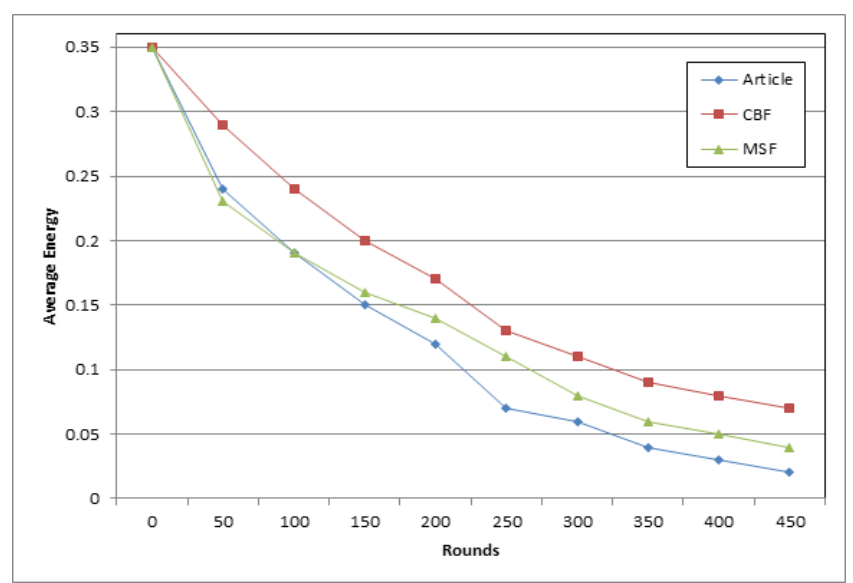

Figure 21. Comparison average energy Vs three algorithm 


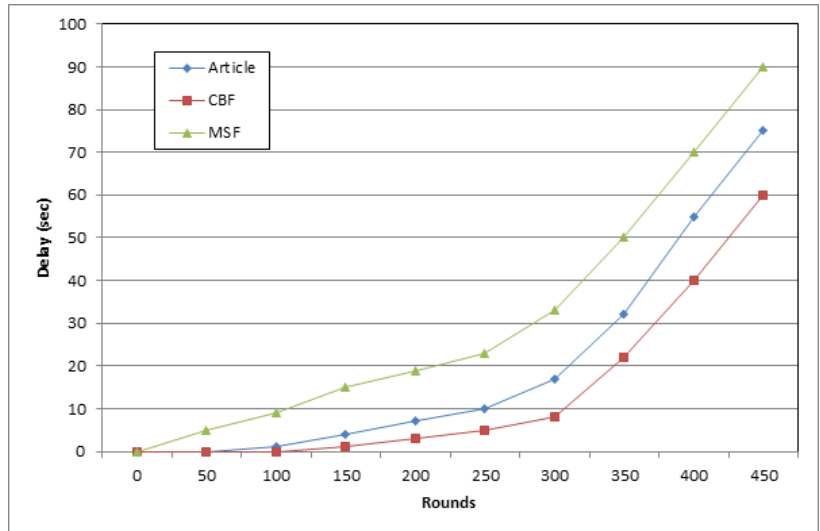

Figure 22. Comparison Delay Vs three algorithm

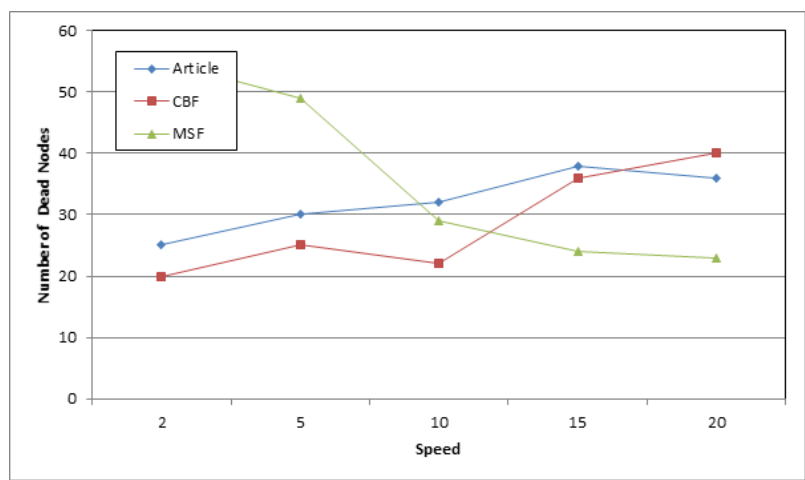

Figure 23. The impact of speed on remaining energy Vs three algorithms

Figure (21), (22), (23) shows the simulation results of CBF and MSF in event-driven scenarios. CBF is a good improvement compared to MSF Therefore, the use of fuzzy logic, to choose a meeting place and cluster nodes makes more efficient use of energy in the network.

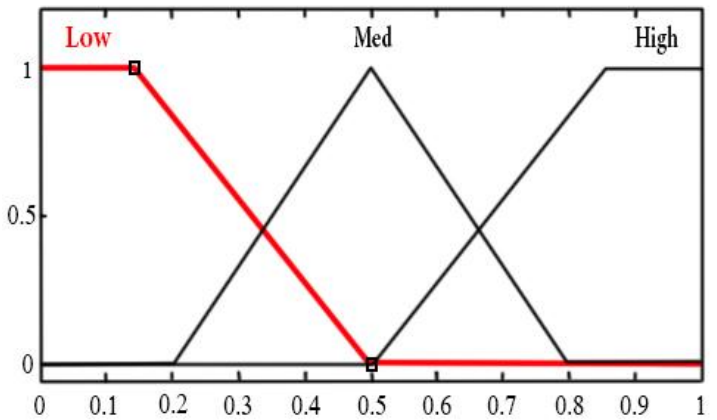

Figure 24. The impact of speed on remaining energy, Vs 300 process of for different speeds sink

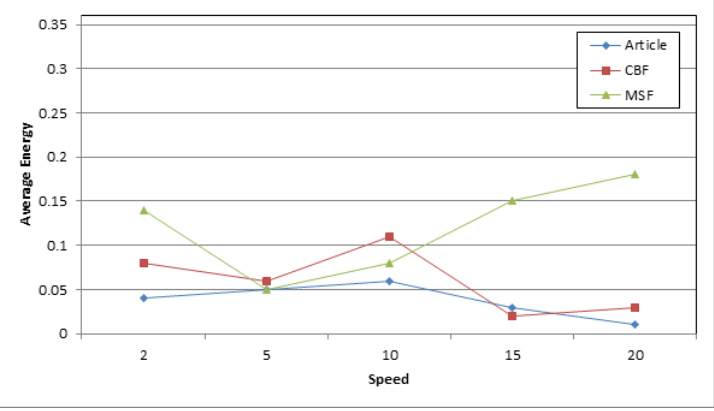

Figure 25. The impact of speed on Average remaining energy, Vs 300 process of for different speeds sink

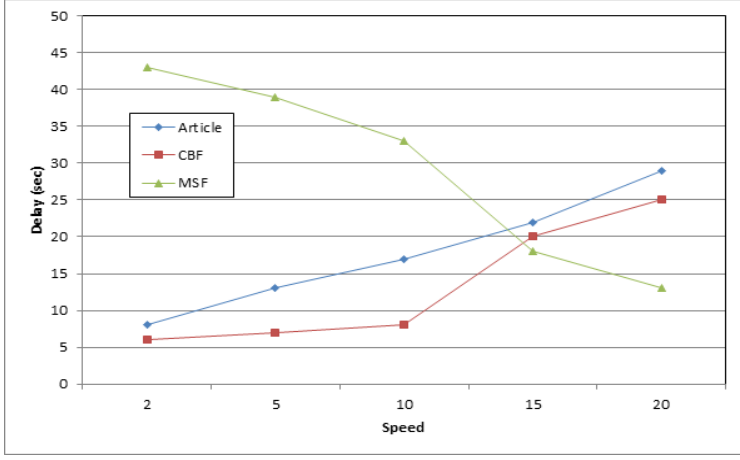

Figure 26. The impact of speed on Delay, Vs 300 process of for different speeds sink

Figure (24),(25),(26). Shows that according to the By increasing the speed sink will have a reduction in delay in the MSF algorithm. The results have indicated that they had a $25 \%$ reduction in delay in network Using Fuzzy Logic to Control the Movement of the Mobile Sink. The MSF algorithm performs better, extends several rounds more than the other algorithm for data transmission, and saves more energy.

\section{CONCLUSION}

An important issue for wireless sensor networks is finding ways to decrease energy consumption. The present study proposed an algorithm that combines the concepts of a mobile sink for decrease delay and average consumption average energy. Here are two methods were presented to collect data on wireless sensor networks. In the first method, called MSF, presents a method for routing the moving sink in wireless sensor networks based on the fuzzy logic. In MSF, it was assumed that a moving sink can move on the network and has unlimited energy. The main issue is the routing of this moving sink so that the lowest energy is consumed for data transfer from the cluster heads to be moving sink.

In the second method, which is called as CBF, the route of the sink has been predetermined and the sink is commuting on this route. There are three types of nodes in the network in the CBF method, ordinary nodes, $\mathrm{RN}$ nodes and $\mathrm{CH}$ nodes. $\mathrm{RN}$ nodes are selected based on a fuzzy system. The nodes are in a close distance from the route of the moving sink, and the cluster nodes use these nodes to transfer information to the sink. The member nodes calculate a priority for themselves using these two parameters of this fuzzy system. Then send the priority to their cluster head along with the message of transferring the information, and then the current cluster head selects the next cluster head for based on this priority from among the member nodes of their cluster. simulation result by omnet++ show that the proposed algorithm in measure delay, consummation average energy and remained energy performs better.

\section{REFERENCES}

[1] F. Zhao and L. Guibas, Wireless Sensor Networks: An Information Processing Approach. Morgan Kaufmann, 2004.

[2] M.A. Batalin, G.S. Sukhatme, M. Hattig, Mobile robot navigation using a sensor network, in: Proc. of IEEE Int'l Conference on Robotics and Automation (ICRA), pp. 636-641, 2004.

[3] P. Guo, T. Jiang, Q. Zhang, K. Zhang, Sleep scheduling for critical event monitoring in wireless sensor networks, IEEE Transactions on Parallel Distributed System 23 (2) (2012) 345-352.

[4] C.-Y. Lin, W.-C. Peng, Y.-C. Tseng, Efficient in-network moving object tracking in wireless sensor networks, 
IEEE Transactions on Mobile Computing 5 (8) (2006) 1044-1056.

[5] Y. Xu, J. Winter, W.-C. Lee, Prediction-based strategies for energy saving in object tracking sensor networks, in: Proc. of IEEE Int'l Conference on Mobile Data Management (MDM), pp. 346-357, 2004.

[6] Terrestrial ecology observing systems. <http://research.cens.ucla.edu/areas/ 2007/Terrestrial/>

[7] R. Szewczyk, A. Mainwaring, J. Polastre, J. Anderson, D. Culler, An analysis of a large scale habitat monitoring application, in: Proc. of ACM Int'l Conference on Embedded Networked Sensor Systems (SenSys), pp. 214-226, 2004.

[8] S. Gandham, Y. Zhang, Q. Huang, Distributed timeoptimal scheduling for convergecast in wireless sensor networks, Computer Networks 52 (3) (2008) 610-629.

[9] O.D. Incel, A. Ghosh, B. Krishnamachari, K. Chintalapudi, Fast data collection in tree-based wireless sensor networks, IEEE Transactions on Mobile Computing 11 (1) (2012) 86-99.

[10] G. Lu, B. Krishnamachari, C.S. Raghavendra, An adaptive energy-efficient and low-latency MAC for treebased data gathering in sensor networks, Wireless Commununications and Mobile Computing (WCMC) 7 (7) (2007) 863-875.

[11] B. Malhotra, I. Nikolaidis, M.A. Nascimento, Aggregation convergecast scheduling in wireless sensor networks, ACM/Kluwer Wireless Networks 17 (2) (2011) 319-335.

[12] S. Upadhyayula, V. Annamalai, S.K.S. Gupta, A lowlatency and energy-efficient algorithm for convergecast in wireless sensor networks, in: Proc. of IEEE Global Telecommunications Conference (Globecom), pp. 35253530, 2003.

[13] G. Lu, B. Krishnamachari, C.S. Raghavendra, An adaptive energy-efficient and low-latency MAC for treebased data gathering in sensor networks, Wireless Commununications and Mobile Computing (WCMC) 7 (7) (2007) 863-875.
[14] B. Malhotra, I. Nikolaidis, M.A. Nascimento, Aggregation convergecast scheduling in wireless sensor networks, ACM/Kluwer Wireless Networks 17 (2) (2011) 319-335.

[15] Wendi Rabiner Heinzelman, Anantha Chandrakasan, and Hari Balakrishnan, "Energy-Efficient Communication Protocol forWireless Microsensor Networks", Proceedings of the 33rd Hawaii International Conference on System Sciences-Volume 8, Page: 8020, 2000

[16] Wendi B. Heinzelman et al., "An Application-Specific Protocol Architecture for Wireless Micro sensor Networks", IEEE transactions on wireless communications, vol. 1, no. 4, October 2002.

[17] Sushant Jain, Rahul C.Shan, et al, "Exploiting Mobility for Energy Efficient Data Collection in Wireless Sensor Networks," Mobile Networks and Applications, vol. 11, no. 3, pp. 327-39, Jun. 2006.

[18] Xinxin Liu, Han Zhao, et al, "Trailing Mobile Sinks: A Proactive Data Reporting Protocol for Wireless Sensor Networks," IEEE Transactions on Computers, pp. 214 223, 2011.

[19] W.R. Heinzelman, A. Chandrakasan, and H. Balakrishnan, "Energy-efficient Communication Protocol for Wireless Microsensor Networks", in IEEE Computer Society Proceedings of the Thirty Third Hawaii International Conference on System Sciences (HICSS '00), Washington, DC, USA, Jan. 2000, vol. 8, pp. 8020.

[20] W.R. Heinzelman, A. Chandrakasan, and H. Balakrishnan, "An Application-Specific Protocol Architecture for Wireless Microsensor Networks" in IEEE Tmnsactions on Wireless Communications (October 2002), vol. 1(4), pp. 660-670.

[21] Lan Wang and Yang Xiao, "A Survey of EnergyEfficient Scheduling Mechanisms in Sensor Network".

[22] S. Singh, M. Woo, and C.S. Raghavendra. "Power-aware routing in mobile ad hoc networks". In Proceedings of the 4th annual ACM/IEEE international conference on Mobile computing and networking (MobiCom), pages 181-190, 1998 